\title{
Demonstration of a Micro-Services Based Multi-Purpose Sensor Platform for Supporting Ambient Assisted Living Systems
}

\author{
Stefan Wagner \\ Aarhus University \\ Finlandsgade 22 \\ 8200 Aarhus, Denmark \\ sw@eng.au.dk
}

\author{
Esben Hunnerup \\ Aliviate Development \\ Sindalsvej 29 \\ 8240 Risskov, Denmark \\ esben.hunnerup@aliviate.dk
}

\author{
Jorge Miranda \\ Aliviate Development \\ Sindalsvej 29 \\ 8240 Risskov, Denmark \\ jorge.miranda@aliviate.dk
}

\begin{abstract}
This paper demonstrates the CARIOT platform and how it interacts with third party ambient assisted living and telemedicine systems. CARIOT is a multi-purpose ambient sensor platform that supports telehealth, telemedicine, and telecare product integration through both cloud and internet of things (IoT) channels, while also supporting closed-network servers and even local-embedded storage for maximum privacy. The aim of this paper is to demonstrate how the CARIOT platform can seamlessly integrate with third party ambient assisted living and telemedicine systems, including: 1) The CAMI LINKWATCH platform, and 2) the OpenTele platform, both using web-services for integration. Also, the paper demonstrates how CARIOT can integrate with other platforms using a micro-services-based approach combined with open communication protocols.
\end{abstract}

\section{CCS CONCEPTS}

- Applied computing $\rightarrow$ Life and medical sciences $\rightarrow$ Health informatics

\section{KEYWORDS}

Ambient assisted living, telemedicine, eHealth, pervasive health

\section{INTRODUCTION}

Ambient assisted living and telemedicine systems have achieved widespread adoption and are quickly maturing in terms of delivering enhanced features and support-services, increasing the efficiency and effectiveness of caregivers, and enhancing citizen self-care. As part of this maturation process, a need exists for supporting new features and services without replacing the already installed hardware and software platforms used by caregiverorganizations. One of the widely implemented telemedicine systems in Denmark is the OpenTele system developed in Denmark as a national reference and open source implementation of telemedicine [1,2]. As an example, the standard telemedicine features found in OpenTele are not sufficient to support the new generation of context-aware self-care systems that are emerging [3]. OpenTele was designed to be open for integration with third party sensor platforms, thus allowing it to be extendable. Also, the CAMI LINKWATCH platform which is an emerging cloud-based platform for assisted living is likewise designed to integrate with third-party sensor platforms [4]. The aim of this paper is to demonstrate how the CARIOT platform can seamlessly integrate with ambient assisted living and telemedicine systems, including: 1) The CAMI LINKWATCH platform, and 2) the OpenTele platform, using regular web-service interfaces. Also, the paper demonstrates how CARIOT can integrate with other platforms using a micro-services-based approach combined with open communication protocols.

\section{METHODS}

\subsection{CARIOT platform}

The CARIOT platform, developed by Aliviate and Ecotopias, enables non-internet connected healthcare devices such as blood pressure, saturation, and weight devices used for traditional telemedicine and telehealth applications, to become internet-ofthings (IoT) enabled. This include support for the open source "The Things Network" IoT Cloud platform, as well as support for commercial IoT platforms such as "Loriot". This integration is achieved using 4G, LoraWAN, and/or WiFi communication channels, depending on the specific usage-scenario.

Several healthcare devices are already supported, and the CARIOT platform can easily be extended with additional healthcare devices. Besides telehealth and telemedicine devices and services, the CARIOT platform supports a range of telecare and personalized medicine devices and services. These include traditional telecare and ambient assisted living services such as medication adherence tracking, fall prevention and detection, and sleep quality, but also generalized support for tracking user activity-levels and activities of daily living using both wearables and ambient sensors [4]. 


\subsection{The OpenTele platform}

The OpenTele platform is an open source telemedicine ecosystem $[1,2]$. According to the $4 \mathrm{~S}$ organization, OpenTele is: "a complete telemedical platform for handling patient recorded outcome data and measurements from personal health devices. The platform comprises a server part and a tablet app installed on a tablet located in the home of the citizen. Using this tablet, the citizen can answer questionnaires, perform measurements and exercises, and communicate with the general practitioner or hospital staff. The server exposes a web portal where a clinician can handle - and communicate with - patients and review the data they collected." [1]. OpenTele is open source, component-based, and highly adaptable and extendable [2]. It is built using the Java programming language for the server side and HTML5 and JavaScript for the web clients (Figure 1). The database is based on the popular MySQL database server, while a REST interface is provided for integration with third party systems using JSON (Java Script Object Notation) encoding [2].

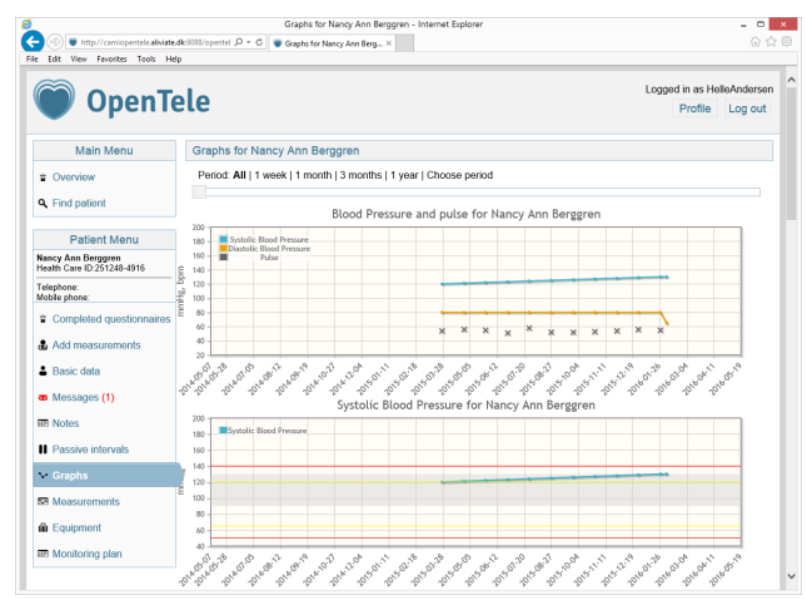

Figure 1. The OpenTele open source telemedicine platform.

\subsection{The CAMI and LINKWATCH platforms}

CAMI is a "Companion with Autonomously Mobile Interface" in an "Artificially intelligent ecosystem for self-management and sustainable quality of life in Ambient Assisted Living". CAMI aims at creating a framework relying on Artificial Intelligence (AI) and allowing a seamless integration of any number of sensors and home appliances with commercial robotic platforms [4]. CAMI's aim is to pave the road of "robots" into the field of ambient assisted living and in settings characterized by a substantial human-machine interaction. CAMI offers a fully integrated AAL solution at the overlap of telecare and telehealth, smart homes and robotics by offering services for social care, home care, and mobility. The LINKWATCH platform is one of several web-based interfaces supported by CAMI allowing caregivers and end-users to access the CAMI platform data and receive notifications from the decision support engine of CAMI (Figure 2).

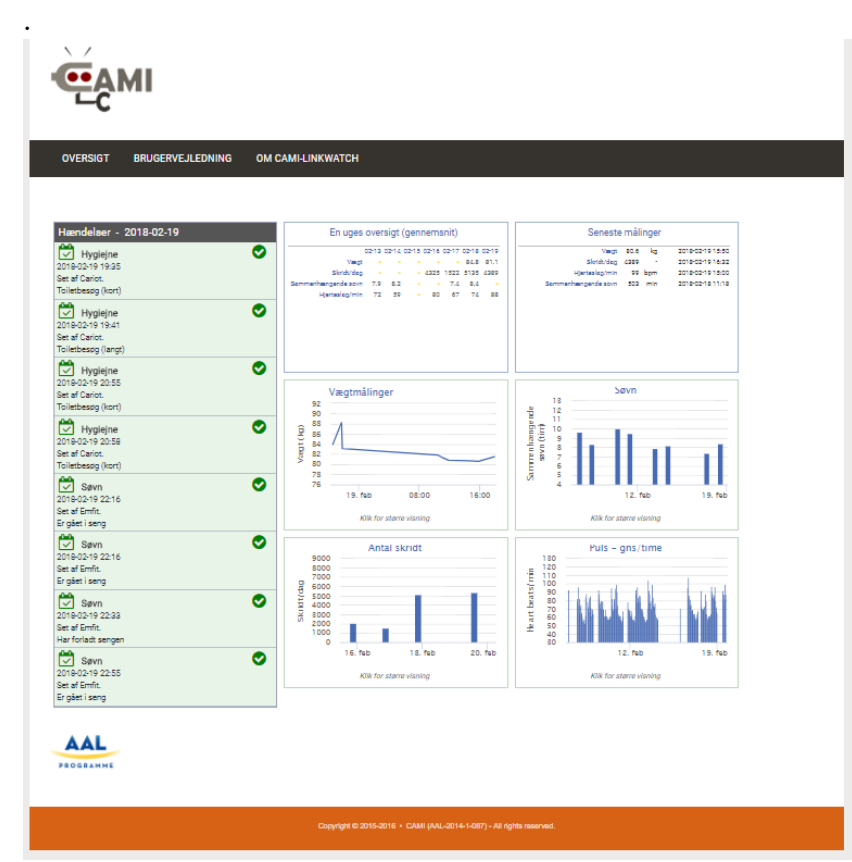

Figure 2. The CAMI LINKWATCH web site showcasing data from the CAMI cloud-based platform, generated by the CARIOT platform. CAMI supports several interfaces.

\section{PLANNED DEMONSTRATION}

A range of CARIOT's services will be demonstrated, including medication adherence, activity, and dehydration trackers, fall detection, as well as dental and body hygiene. Also, CARIOT's medico device integration features will be demonstrated using a blood pressure device and an oximeter. The demonstration will showcase how CARIOT can send data using either $4 \mathrm{G}$, WiFi or LoraWAN radio communications to the OpenTele and CAMI LINKWATCH systems. Also, CARIOT's ability to store data locally in a secure manner, avoiding cloud-based storage scenarios, will be demonstrated.

\section{ACKNOWLEDGMENTS}

Under the project number AAL-2014-1-087, this work was supported within the Active and Assisted Living Programme (AAL) by a grant of the Danish Agency for Science, Technology and Innovation, and co-founded by the company Aliviate and the end-user organization ECOTOPIAS and with support from the CAMI consortium [3].

\section{REFERENCES}

[1] 4S organization. OpenTele. Retrieved April 5th 2017 from http://4sonline.dk/wiki/doku.php?id=opentele: overview

[2] Wagner, S., 2017. Telemedicine with OpenTele. Medivate Publishing.

[3] Memon, M.; Wagner, S.R.; Pedersen, C.F.; Beevi, F.H.A.; Hansen, F.O. Ambient assisted living healthcare frameworks, platforms, standards, and quality attributes. Sensors $2014,14,4312-4341$.

[4] The CAMI consortium. Retrieved March 27th 2018 from http://www.camiproject.eu/ 